\begin{tabular}{|l|l|}
\hline Journal Code: JZO & Proofreader: Mony \\
\hline Article No: JZO12199 & Delivery date: 03 Dec 2014 \\
\hline Page Extent: 3 & \\
\hline
\end{tabular}

EDITORIAL

\title{
Cognitive demands of sociality: from simple mechanisms to complex behaviour
}

\author{
N. Raihani \\ Department of Genetics, Evolution and Environment, University College London, London, UK
}

2 doi:10.1111/jzo.12199

Correspondence

Nichola Raihani, Department of Genetics, Evolution and Environment, University College London, Darwin Building, London WC1E 6BT, UK. Email: nicholaraihani@gmail.com

Individual learning and behaviour are often shaped by the actions of others. For example, individuals might acquire information from others about their environment (Rendell et al., 2010; Leadbeater, ••) while experienced individuals might teach naive counterparts how to behave in a given context (Thornton \& Raihani, 2008). Furthermore, individuals might cooperate with others to achieve personal or common goals, or compete with them over limiting resources (McAuliffe \& Thornton, ••; Thornton \& McAuliffe, ••). Despite the vast and fruitful body of research into these topics, a key outstanding question is whether social learning and behaviour rely on specialized, higher-order cognitive adaptations, either for social learning or for social behaviour more generally. It was with this broad question in mind that this mini-series of reviews was commissioned. The three contributions ask, respectively, whether social learning is an adaptation to social living (Leadbeater, $\bullet$ ), whether cooperative breeding selects for advanced socio-cognitive abilities (Thornton \& McAuliffe, $\bullet$ ), and more generally, how considering both psychology and ecology is essential to understand the mechanisms underpinning social learning and behaviour (McAuliffe \& Thornton, $\bullet$ ). A key take-home message from all three contributions is that seemingly complex behavioural and learning outcomes can often arise through relatively simple psychological mechanisms and, relatedly, that similar behavioural outcomes can often be achieved via different cognitive means.

The tendency to attribute higher-order cognitive abilities to animals that learn and behave socially has, in part, stemmed from comparisons with humans, who are thought to possess and use advanced social cognition to navigate their social world. For example, some forms of human teaching require the teacher to 'know what the pupil knows' (i.e. possess theory of mind) which is a putatively uniquely human capacity (Thornton \& Raihani, 2008; Thornton \& McAuliffe, 2012). Similarly, the human willingness to voluntarily share resources with others (see Engel, 2011) or to punish cheats (Raihani \& McAuliffe, 2012) are both thought to stem from intrinsic fairness preferences (Fehr \& Schmidt, 1999), which rely on the ability to compare own payoffs with those of a social partner. It is perhaps natural, therefore, to ask whether apparently related behaviours in other species might also rely on similar cognitive mechanisms. A key emphasized by the commissioned reviews, however, is that behaviours that serve similar functions need not all rely on the same cognitive mechanisms. For example, although human punishment is thought to be motivated, at least in part, by inequity aversion (Raihani \& McAuliffe, 2012), the bluestreak cleaner wrasse Labroides dimidiatus shows no evidence for fairness preferences (Raihani et al., 2012a,b) despite being known to punish social cheats (e.g. Raihani, Grutter \& Bshary, 2010; Raihani et al., 2012a,b). Similarly, although some forms of human teaching might use - or even rely on - theory of mind, examples of teaching in both humans and non-humans exist that do not necessitate such complex cognition (e.g. Thornton \& McAuliffe, 2012). For example, a meerkat helper can teach a pup by provisioning it with increasingly live prey items, giving the pup opportunities to learn handling skills. However, this behaviour need not rely on the helper 'knowing what the pup knows'. Instead, experimental evidence indicates that helpers adjust provisioning behaviour according to the pup's begging calls which serve as a reliable proxy for age and therefore prey-handling expertise (Thornton \& McAuliffe, 2006). These examples highlight the importance of distinguishing between behavioural function, on the one hand, and proximate mechanism on the other (Scott-Phillips, Dickins \& West, 2011; Thornton \& Raihani, in press). Behaviour with similar evolutionary functions (e.g. promoting learning or cooperation) might occur via markedly different mechanisms - and functions should therefore not be defined according to mechanisms (Thornton \& Raihani, in press). Instead, it may be more helpful to ask why - from an ultimate perspective - some species use cognitively complex psychological mechanisms to achieve outcomes where simpler and less demanding mechanisms might often suffice.

A related point is that ignoring ecology may lead to dubious inferences about mechanisms underpinning behaviour. For example, as pointed out by McAuliffe \& Thornton $(\bullet)$, the 
tendency for rats to release trapped conspecifics in laboratory settings has been argued as being motivated by psychological empathy (e.g. Bartal, Decety \& Mason, 2011), but this inference appears problematic when considering that, in the wild, rats routinely attack and kill unfamiliar individuals (Blanchard et al., 1975). Similar points have been raised regarding the interpretation of active food sharing in nonhuman primates in laboratory studies as indicative of otherregarding or fairness preferences (e.g. Burkart et al., 2007; Horner, Carter \& Suchak, 2011). As McAuliffe \& Thornton $(\bullet)$ argue, these behaviours are often rare under natural circumstances and, when they occur, often reflect self-interested responses to harassment rather than psychologically altruistic motives to help others (Gilby, 2006). These examples underline the importance of considering whether the ecology of the animal in question is likely to have selected for prosociality before inferring that animals have cognitive mechanisms that are adapted to helping others.

Meanwhile, others have also asked whether possession of common cognitive abilities might result in similar outcomes. For example, the tendency to observe and copy the behaviour of others (social learning) underpins the emergence of grouptypical norms of behaviour or culture (Boyd \& Richerson, 1985). Given that several non-human species learn socially, it is perhaps unsurprising that there is also strong evidence for culture in many non-human species (Levebre \& Palameta, 1988; Whiten et al., 1999; Rendell \& Whitehead, 2001; Laland $\&$ Hoppitt, 2003). Nevertheless, as Leadbeater ( $\bullet$ ) points out, culture is not an inevitable outcome of the ability to learn socially. Indeed, cumulative culture [where cultural modifications accumulate over time and build on previous advances (Tomasello, Kruger \& Ratner, 1993)] is thought to be unique to humans (Tennie, Call \& Tomasello, 2009 but see 4 Yamamoto, Humle \& Tanaka, 2013). Given that several nonhuman species have the cognitive machinery to facilitate the emergence of culture and even, arguably, cumulative culture (Dean et al., 2012; Leadbeater, ••), why are these phenomena not more widespread? Only approaches that consider both ecology and psychology in tandem are likely to be able to answer these sorts of questions.

\section{References}

Bartal, L.B.-A., Decety, J. \& Mason, P. (2011). Empathy and pro-social behavior in rats. Science 334, 1427-1430.

Blanchard, R.J., Fukunaga, K., Blanchard, D.C. \& Kelley, M.J. (1975). Conspecific aggression in the laboratory rat. $J$. Comp. Physiol. Psychol. 89, 1204-1209.

Boyd, R. \& Richerson, P. (1985). Culture and the evolutionary process. Chicago: University of Chicago Press.

Burkart, J.M., Fehr, E., Efferson, C. \& van Schaik, C. (2007). Other-regarding preferences in a non-human primate: common marmosets provision food altruistically. Proc. Natl. Acad. Sci. USA 104, 19762-19766.

Dean, L.G., Kendal, R.L., Schapiro, S.J., Thierry, B. \& Laland, K.N. (2012). Identification of the social and cognitive processes underlying human cumulative culture. Science 335, 1114-1118.

Engel, C. (2011). Dictator games: a meta study. Exp. Econ. 14, 583-610.

Fehr, E. \& Schmidt, K.M. (1999). A theory of fairness, competition, and cooperation. Quart. J. Econ. 114, $817-868$.

Gilby, I. (2006). Meat sharing among the Gombe chimpanzees: harassment and reciprocal exchange. Anim. Behav. 71, 953-963.

Horner, V., Carter, J. \& Suchak, M. (2011). Spontaneous prosocial choice by chimpanzees. Proc. Natl. Acad. Sci. USA 108, 13847-13851.

Laland, K.N. \& Hoppitt, W. (2003). Do animals have culture? Evol. Anthropol. 12, 150-159.

Leadbeater, $\bullet$. $(\bullet \bullet)$. What evolves in the evolution of social learning? J. Zool. (Lond.) ••, •••••.

Levebre, L. \& Palameta, B. (1988). Mechanisms, ecology, and population diffusion of socially-learned, food-finding behavior in feral pigeons. In Social learning, psychological and biological perspectives: 141-165. Zentall, T. \& Galef, B.G. Jr. (Eds). Hillsdale: Lawrence Erlbaum Associates, Inc.

McAuliffe, $\bullet$ \& Thornton, $\bullet(\bullet \bullet)$. The psychology of cooperation in animals: an ecological approach. J. Zool. (Lond.) $\bullet, \cdot \bullet \cdot \bullet$.

Raihani, N.J. \& McAuliffe, K. (2012). Human punishment is motivated by inequity aversion, not a desire for reciprocity. Biol. Lett. 8, 802-804.

Raihani, N.J., Grutter, A.S. \& Bshary, R. (2010). Punishers benefit from third-party punishment in fish. Science 327, 171.

Raihani, N.J., McAuliffe, K., Brosnan, S.F. \& Bshary, R. (2012a). Are cleaner fish, Labroides dimidiatus, inequity averse? Anim. Behav. 84, 665-674.

Raihani, N.J., Pinto, A.I., Grutter, A.S., Wismer, S. \& Bshary, R. (2012b). Male cleaner wrasses adjust punishment of female partners according to the stakes. Proc. Roy. Soc. Lond. Ser. B. 279, 365-370.

Rendell, L. \& Whitehead, H. (2001). Culture in whales and dolphins. Behav. Brain Sci. 24, 309-324.

Rendell, L., Laland, K.N., Boyd, R., Cownden, D., Enquist, M., Eriksson, K., Feldman, M.W., Fogarty, L., Ghirlanda, S. \& Lillicrap, T. (2010). Why copy others? Insights from the social learning strategies tournament. Science 328, 208213.

Scott-Phillips, T.C., Dickins, T.E. \& West, S.A. (2011). Evolutionary theory and the ultimate-proximate distinction in the human behavioral sciences. Perspect. Psychol. Sci. 6, 38-47.

Tennie, C., Call, J. \& Tomasello, M. (2009). Ratcheting up the ratchet: on the evolution of cumulative culture. Philos. Trans. Roy. Soc. B. 364, 2405-2415.

Thornton, A. \& McAuliffe, K. (••). Cognitive consequences of cooperative breeding? A critical appraisal. J. Zool. (Lond.) $\bullet, \cdot \cdots \cdot \cdot$ 
Thornton, A. \& McAuliffe, K. (2006). Teaching in wild meerkats. Science 313, 227-229.

Thornton, A. \& McAuliffe, K. (2012). Teaching can teach us a lot. Anim. Behav. 83, e6-e9.

Thornton, A. \& Raihani, N.J. (2008). The evolution of teaching. Anim. Behav. 75, 1823-1836.

Thornton, A. \& Raihani, N.J. (in press). The proximateultimate confusion in teaching and cooperation. Behav. Brain Sci. $\bullet \bullet, \bullet \bullet \cdot \bullet$.

Tomasello, M., Kruger, A.C. \& Ratner, H.H. (1993). Cultural learning. Behav. Brain Sci. 16, 495-511.
Tomasello, $\bullet$. (1994). $\bullet$.

Whiten, A., Goodall, J., McGrew, W.C., Nishida, T., Reynolds, V., Sugiyama, Y., Tutin, C.E.G., Wangham, R.W. \& Boesch, C. (1999). Cultures in chimpanzees. Nature 399, 682-685.

Yamamoto, S., Humle, T. \& Tanaka, M. (2013). Basis for cumulative cultural evolution in chimpanzees: social learning of a more efficient tool-use technique. PLoS ONE 8, e55768. 


\section{AUTHOR QUERY FORM}

Dear Author,

During the preparation of your manuscript for publication, the questions listed below have arisen. Please attend to these matters and return this form with your proof.

Many thanks for your assistance.

\begin{tabular}{|c|c|c|}
\hline Query References & Query & Remarks \\
\hline 1 & $\begin{array}{l}\text { AUTHOR: Please confirm that given names (red) and surnames/family names } \\
\text { (green) have been identified correctly. }\end{array}$ & \\
\hline 2 & WILEY BLACKWELL: Please provide the doi number for this article. & \\
\hline 3 & $\begin{array}{l}\text { AUTHOR: For Raihani et al. 2012, more than one reference shares the same } \\
\text { author and year-of-publication details. Please check that they have been correctly } \\
\text { differentiated between using a, b, etc. after the year of publication in the reference } \\
\text { citation and the reference list. }\end{array}$ & \\
\hline 4 & $\begin{array}{l}\text { AUTHOR: Please check if the parentheses and brackets were used correctly in the } \\
\text { sentence 'Indeed . . to humans.' }\end{array}$ & \\
\hline 5 & $\begin{array}{l}\text { AUTHOR: 'Given . . widespread?' This sentence has been modified for clarity. } \\
\text { Please check if this is correct. }\end{array}$ & \\
\hline 6 & $\begin{array}{l}\text { AUTHOR: Please check if the abbreviated journal title for Reference Fehr \& } \\
\text { Schmidt } 1999 \text { is correct. }\end{array}$ & \\
\hline 7 & AUTHOR: Please supply the initials for Reference Leadbeater. & \\
\hline 8 & $\begin{array}{l}\text { WILEY BLACKWELL: Please update the year of publication, volume number } \\
\text { and page range for Reference Leadbeater. }\end{array}$ & \\
\hline 9 & $\begin{array}{l}\text { WILEY BLACKWELL: Please update the year of publication, volume number } \\
\text { and page range for Reference McAuliffe \& Thornton. }\end{array}$ & \\
\hline 10 & AUTHOR: Please provide the initials of author McAuliffe and Thornton. & \\
\hline 11 & $\begin{array}{l}\text { WILEY BLACKWELL: Please update the year of publication, volume number } \\
\text { and page range for Reference Thornton \& McAuliffe. }\end{array}$ & \\
\hline 12 & $\begin{array}{l}\text { AUTHOR: Please update the year of publication, volume number and page range } \\
\text { for Thornton and Raihani, in press. }\end{array}$ & \\
\hline 13 & $\begin{array}{l}\text { AUTHOR: Please provide the initials of author Tomasello and the document title } \\
\text { for Tomasello 1994. Note also that Tomasello } 1994 \text { has not been cited in the text. } \\
\text { Please indicate where it should be cited; or delete from the reference list. }\end{array}$ & \\
\hline
\end{tabular}


Required software to e-Annotate PDFs: Adobe Acrobat Professional or Adobe Reader (version 8.0 or above). (Note that this document uses screenshots from Adobe Reader $\mathbf{X}$ )

The latest version of Acrobat Reader can be downloaded for free at: http://get.adobe.com/reader/

Once you have Acrobat Reader open on your computer, click on the Comment tab at the right of the toolbar:

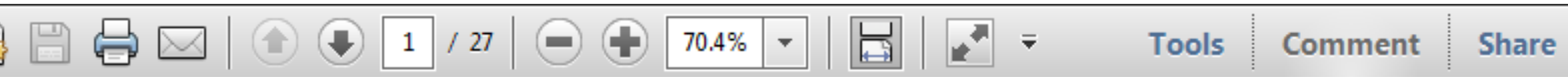

This will open up a panel down the right side of the document. The majority of tools you will use for annotating your proof will be in the Annotations section, pictured opposite. We've picked out some of these tools below:

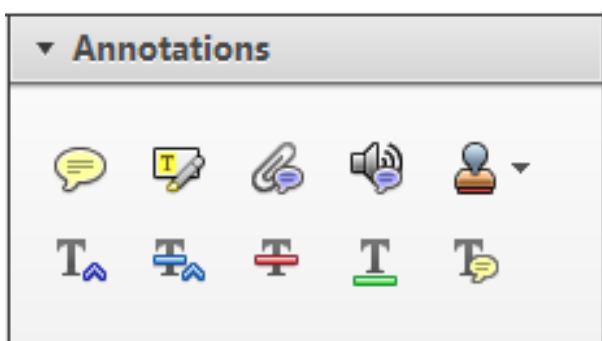

1. Replace (Ins) Tool - for replacing text.

Strikes a line through text and opens up a text box where replacement text can be entered.

\section{How to use it}

- Highlight a word or sentence.

- Click on the Replace (Ins) icon in the Annotations section.

- Type the replacement text into the blue box that appears.

Idard tramework for the analysis of $\mathrm{m}$ icy Nevertheless, it also led to exog،

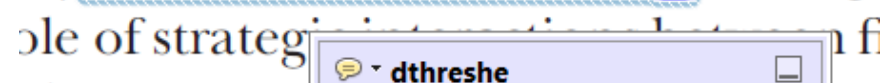
aber of comp 08/06/2011 15:58:17 $\quad$ o is that the $\mathrm{s} 1$, which led of nain compo: be level, are exc nc

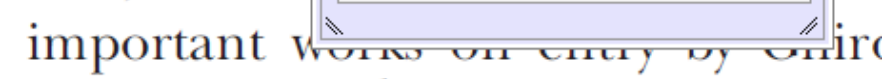
M heneforth) ${ }^{1}$ we snen the 'hlarl $\mathrm{h}$

3. Add note to text Tool - for highlighting a section to be changed to bold or italic.

Tighlights text in yellow and opens up a text box where comments can be entered.

\section{How to use it}

- Highlight the relevant section of text.

- Click on the Add note to text icon in the Annotations section.

- Type instruction on what should be changed regarding the text into the yellow box that annears.

namic responses of mark ups ent with the VAR evidence

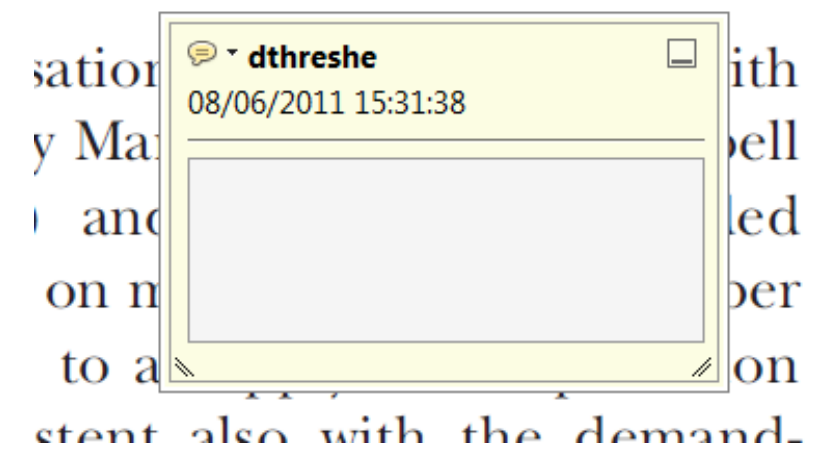

2. Strikethrough (Del) Tool - for deleting text.

Thikes a red line through text that is to be deleted.

\section{How to use it}

- Highlight a word or sentence.

- Click on the Strikethrough (Del) icon in the Annotations section.

there is no room for extra protits al c ups are zero and the number of ret) values are not determined by Blanchard and Kiyotaki (1987), sfect competition in general equilil ts of aggregate demand and supply lassical framework assuming monol een an evorenous number of firms

\section{Add sticky note Tool - for making notes at} specific points in the text.

\section{Marks a point in the proof where a comment} needs to be highlighted.

\section{How to use it}

- Click on the Add sticky note icon in the Annotations section.

- Click at the point in the proof where the comment should be inserted.

- Type the comment into the yellow box that appears.

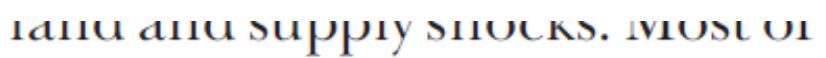

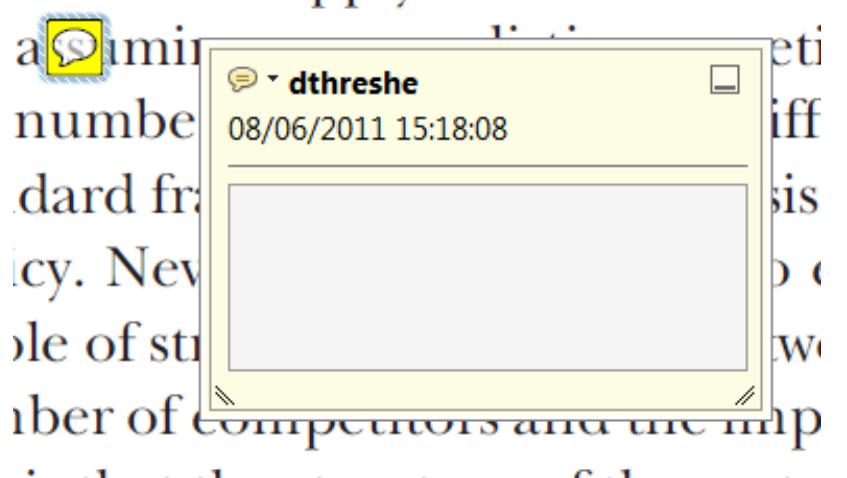

is that the structure of the secto 
5. Attach File Tool - for inserting large amounts of text or replacement figures.

Inserts an icon linking to the attached file in the appropriate pace in the text.

\section{How to use it}

- Click on the Attach File icon in the Annotations section.

- Click on the proof to where you'd like the attached file to be linked.

- Select the file to be attached from your computer or network.

- Select the colour and type of icon that will appear in the proof. Click OK.

E N D

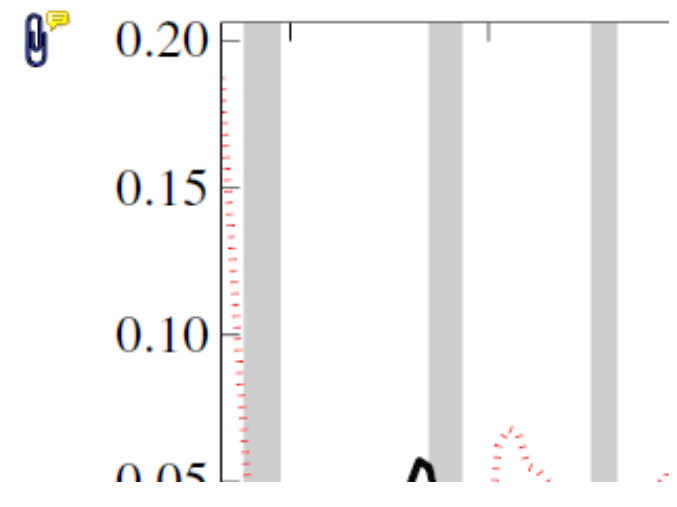

6. Add stamp Tool - for approving a proof if no corrections are required.

- Inserts a selected stamp onto an appropriate place in the proof.

\section{How to use it}

- Click on the Add stamp icon in the Annotations section.

- $\quad$ Select the stamp you want to use. (The Approved stamp is usually available directly in the menu that appears).

- Click on the proof where you'd like the stamp to appear. (Where a proof is to be approved as it is, this would normally be on the first page).

of the Dusiness cycie, starting with the on perfect competition, constant ret

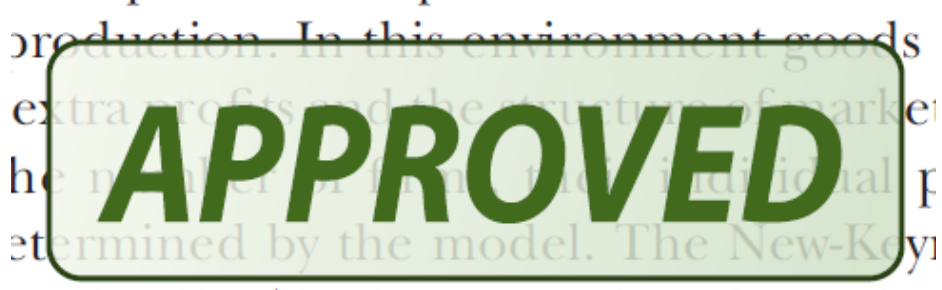

otaki (1987), has introduced produc general equilibrium models with nomin
Drawing Markups

藏 可 $\Rightarrow$

$0 \square \sqrt{6} D$

\section{How to use it}

- Click on one of the shapes in the Drawing Markups section.

- Click on the proof at the relevant point and draw the selected shape with the cursor.

- To add a comment to the drawn shape, move the cursor over the shape until an arrowhead appears.

- Double click on the shape and type any text in the red box that appears.
7. Drawing Markups Tools - for drawing shapes, lines and freeform annotations on proofs and commenting on these marks.

Allows shapes, lines and freeform annotations to be drawn on proofs and for comment to be made on these marks.

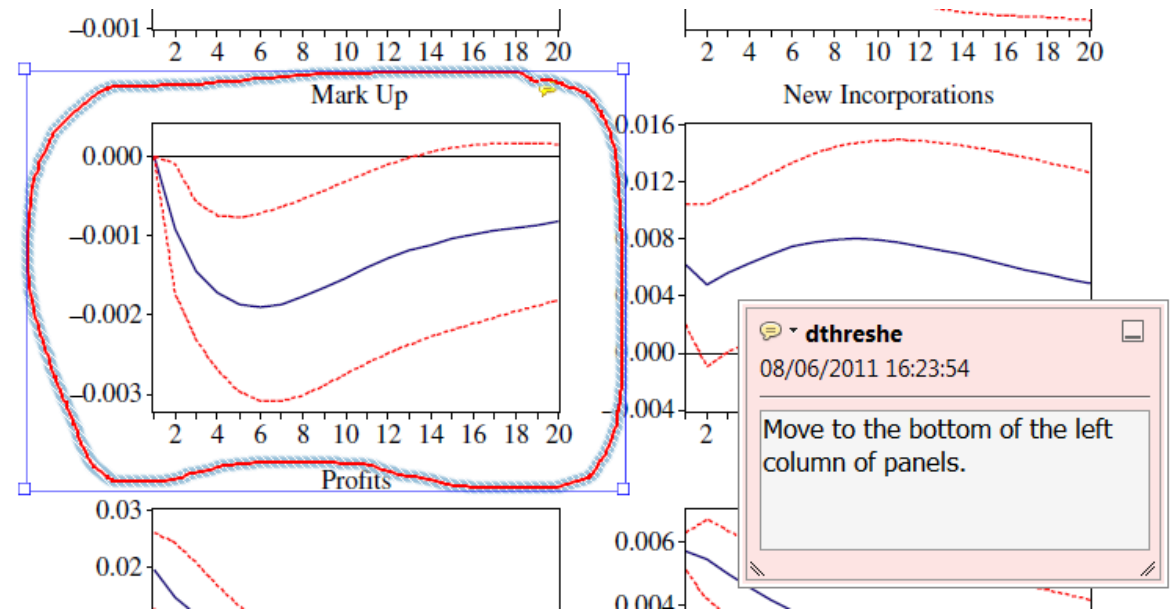

For further information on how to annotate proofs, click on the Help menu to reveal a list of further options:

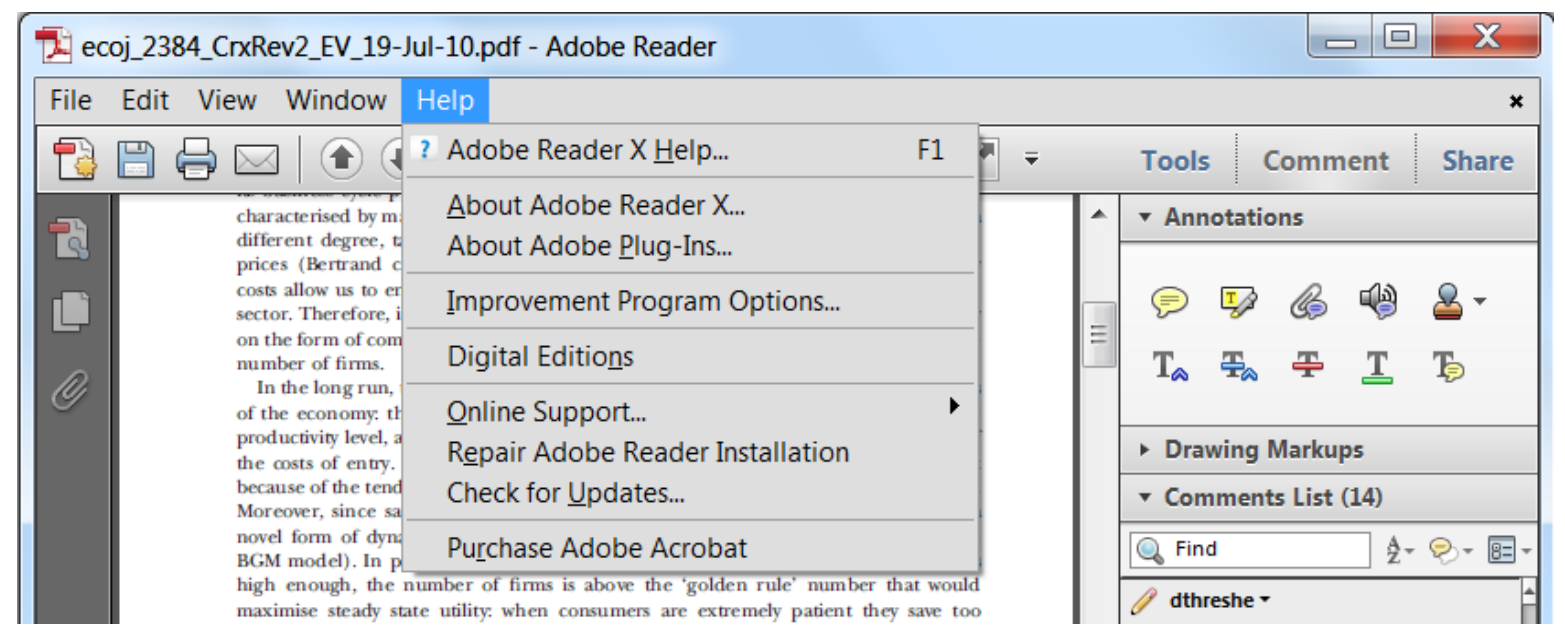

